\title{
PATHOLOGICAL PARAMETERS PREDICTING HER-2/NEU STATUS OF BREAST CARCINOMA
}

\author{
L Mudduwa \\ Department of Pathology, Faculty of Medicine, Galle
}

\begin{abstract}
Introduction: Immunohistochemical detection of HER-2/neu expression in breast carcinoma is a required test, yet expensive. Only $10-30 \%$ of breast cancers over-express HER-2/neu. Therefore it is important to evaluate the pathological parameters which predict HER-2/neu expression in order to select patients for HER-2/neu assessment in a poor resource setting.

Objective: To determine the positive and negative predictive values of Nottingham Grade, Nottingham Prognostic Index (NPI) and estrogen and progesterone receptor (ER and PR) status in predicting the HER-2/neu status of breast carcinoma.

Method: Seventy five consecutive invasive breast carcinomas were assessed for HER-2/neu status at the Diagnostic Immunohistochemistry Laboratory, Department of Pathology, Faculty of Medicine, Galle. Predictive values of the aforementioned four histological parameters were assessed in predicting the HER-2/neu status of breast carcinoma.

Results: Out of the 75 breast carcinomas 18 (24\%) strongly expressed HER-2/neu. A Quick Score of 0 or 2 for ER gave a positive predictive value of $37 \%$ and a negative predictive value of $91 \%$ in predicting HER-2/neu status. The positive and negative predictive values of a Quick Score of 0 or 2 for PR were $32 \%$ and $86 \%$ respectively. The Nottingham grade being 2 or 3 had a positive predictive value of $26 \%$ and a negative predictive value of $91 \%$. NPI of $>3.4$ had a positive predictive value of $26 \%$ and a negative predictive value $93.2 \%$.

Conclusion: Nottingham Grade of 2 or 3, NPI of $>3.4$ and a Quick Score of 0 or 2 for ER and PR have a good negative predictive value in predicting the HER-2/neu status of breast carcinoma. NPI is the best parameter in this respect.
\end{abstract}

Keywords: Breast carcinoma, HER2, ER, PR, NPI, Nottingham grade.

Address for correspondence: L Mudduwa, Department of Pathology, P.O. Box 70, Faculty of Medicine, Galle, Sri Lanka.

e-mail: lakminimudduwa@yahoo.com 


\section{Introduction}

Breast carcinoma is the commonest cancer in women worldwide, as well as in Sri Lanka. ${ }^{1}$ The HER-2/neu (c-erbB2) oncogene was identified from chemical carcinogen ethyl-nitroso-urea induced neuroblastomas of rats. $^{2}$ The human homology of HER-2/neu is present in chromosome 17 and encodes a $185 \mathrm{kd}$ trans-membrane glycoprotein. In breast carcinoma amplification of HER-2/neu and over expression of the HER-2/neu encoded protein are associated with a poor prognosis with a median survival of 3 years compared to 6-7 years for HER-2/neu negative cases. ${ }^{3}$

Trastuzumab (Herceptin ${ }^{\circledR}$ ) is a humanized monoclonal antibody that selectively binds to the extracellular domain of the 185 kd HER-2/neu protein. ${ }^{4}$ It is the first therapy for breast carcinoma which targets an oncogene product. ${ }^{4}$ It has been recommended by the Food and Drug Authority for administration to women with metastatic breast cancer whose tumours express HER-2/neu. This humanized antibody to HER-2/neu has shown to have higher response rate and improved survival. ${ }^{3}$ The value of Trastuzumab therapy for early breast carcinoma is still a research topic. Testing samples for HER-2/neu status is routinely done by immunohistochemistry (IHC), which is a well established method but IHC analysis may be subject to differences in tissue fixation, processing and use of different antibodies. This may render the IHC technique less than completely objective. ${ }^{3,5}$ Fluorescent in-situ hybridization (FISH) has been reported to be the most sensitive technique for quantitative evaluation of HER$2 /$ neu gene status but it is a technique which needs special laboratory facilities and a longer time to carry out. Therefore FISH cannot be done routinely to assess the HER-2/neu status. In a country where the resources are scarce it is important to select patients for assessment of the HER-2 status which is found to be expressed by only $10-30 \%$ of patients. ${ }^{6}$

The aim of this study was to determine the predictive value of pathological parameters which are routinely assessed in reporting breast cancers, to select patients for immunohistochemical assessment of HER-2/neu status. This study intended to determine the positive and negative predictive values of Nottingham Grade, Nottingham Prognostic Index (NPI) and hormone receptor status in predicting the HER-2/neu status of invasive breast carcinoma.

\section{Materials and methods}

This descriptive cross sectional study was carried out at the diagnostic immunohistochemistry laboratory, Department of Pathology, Faculty of Medicine, University of Ruhuna, Sri Lanka. The breast carcinomas assessed for HER-2/neu status by the author for a period of one year from June 2006 were included in the study. Cases reported by the others at the same centre were 
excluded in order to assure consistency in assigning grades and scores. Informed written consent was obtained from each patient before they were enrolled for the study. Prior ethical approval was obtained from the Ethical Review Committee of the Faculty of Medicine, University of Ruhuna, Sri Lanka.

The tumour grade was assessed by the author on the H\&E stained slide using the Nottingham modification of the Bloom and Richardson grading system. ${ }^{7}$ The immunohistochemical staining for HER-2/neu was scored according to the guidelines published by Ellis 10 et al. ${ }^{8}$ Estrogen and progesterone receptor (ER and PR) staining were given a score ranging from 0 to 8 using the Quick Score. ${ }^{9}$ Immunostaining was performed using the Streptavidin Biotin method. Dako ${ }^{\circledR}$ polyclonal rabbit antihuman progesterone receptor (A0098), polyclonal rabbit antihuman cerbB-2 oncoprotein (A048520), Monoclonal mouse antihuman estrogen receptor $\alpha$, clone 1D5 (M704701) and Universal LSAB2 kit/HRP rabbit/mouse with Streptavidin/HRP (K067511, K067589) were used for immunohistochemical staining which was done manually by an experienced technical officer. Sections of 3-4 $\mu$ thickness were taken from paraffin wax blocks for immunostaining.

NPI was calculated using the formula recommended by the UK NHS guidelines. ${ }^{10}$ The sensitivity and specificity of Quick Score of 0 or 2 for ER and PR, Nottingham Grade of 2-3 and NPI of >3.4 in predicting HER-2/neu status were assessed. For this study a Quick Score of 0 or 2 was considered negative staining. A Quick Score of 2 was assigned to breast carcinomas when $<10 \%$ of cells show weak nuclear staining which is said to indicate only a small chance of response to endocrine therapy. ${ }^{9}$ The sensitivity and specificity of each parameter and the prevalence of HER-2/neu were calculated and the positive and negative predictive values were determined using standard formulae.

\section{Results}

Seventy five patients who had HER-2/neu status assessed from June 2006 to June 2007 were included in the study. The histological grade was assessed in all 75 patients on the H\&E sections. ER and PR status were available for 74 patients. NPI was calculated for 67 patients and for eight patients lymph node status was not available and hence the NPI could not be calculated for them.

Eighteen patients (24\%) had a score of +3 and were considered positive for HER-2/neu overexpression. Only two patients had +2 and were considered negative for this study as facilities were not available for FISH. A Quick Score of 2 or less (2 or 0) was observed in 41 (55.4\%) patients for ER and in 40 (54\%) patients for PR. Sixty two patients (82.7\%) had a Nottingham grade of 2 or 3 and 17 of them were HER-2/neu positive. There were only twelve patients with a Nottingham grade of 1 and only one of them was HER-2/neu positive. Out of the 67 patients 
for whom the NPI was calculated 51 (76.1\%) had an NPI of more than 3.4. Out of all HER-2/neu positive patients only three had a NPI of 3.4 or less. Positive and negative predictive values for the four parameters were calculated taking the prevalence of HER-2/neu positivity into account (Table 1).

Table 1. Sensitivity, specificity, positive and negative predictive values of pathological parameters.

\begin{tabular}{|lllll|}
\hline $\begin{array}{l}\text { Pathological } \\
\text { parameter }\end{array}$ & Sensitivity & Specificity & $\begin{array}{l}\text { Positive } \\
\text { Predictive Value }\end{array}$ & $\begin{array}{l}\text { Negative } \\
\text { Predictive Value }\end{array}$ \\
\hline ER Quick Score 0 & 83.3 & 53 & 37 & 91 \\
\hline PR Quick Score 0 & 72.2 & 51.7 & 32 & 86 \\
\hline Grade 2-3 & 94.4 & 21 & 26 & 91 \\
\hline NPI >=3.4 & 83.3 & 26.5 & 26 & 93.2 \\
\hline
\end{tabular}

\section{Discussion and Conclusion}

Age, tumour size, grade, hormone receptor status, HER-2/neu status and lymph node status have been recognized as prognostic indicators of breast carcinoma. Uniform agreement exists that the status of the axillary lymph nodes is the single most important prognostic factor for patients with breast carcinoma. ${ }^{10}$

The presence of ER and PR represent a relatively weak prognostic factor for patients with breast cancer but they are the strongest predictive factors for response to hormone therapy. ${ }^{10}$ The percentage of breast carcinoma patients over-expressing HER-2/neu in the present study was $24 \%$ and it is comparable to that in world literature which ranges from $10-30 \%{ }^{6}$ However the expression of ER and PR appears to be lower. NPI of 3.4 or less was seen in only $23.8 \%$ of patients indicating a good prognosis. The majority had an NPI of $>3.4$. Only three HER-2/neu positive tumours in the study had an NPI of 3.4 or less.

Many studies have shown that HER-2/neu over-expression is an adverse prognostic feature. HER-2/neu over-expressing tumours have shown an increased disease recurrence and metastasis and shortened survival. ${ }^{6}$ Patients with HER-2/neu over-expressing tumours with metastasis are selected for treatment with trastuzumab. Recent studies have shown good response to trastuzumab in patients with node negative breast carcinoma as well. The results of the HERA trial indicate that one year of adjuvant trastuzumab should be considered a standard option on completion of loco-regional therapy and neoadjuvant chemotherapy for women who have node positive disease or node negative disease with tumour size $>1 \mathrm{~cm} .{ }^{11}$ Therefore the usefulness of the assessment of HER-2/neu status is becoming more important. 
The association of HER-2/neu with other prognostic factors has been of great interest. Studies have been done to evaluate the relationship between over expression of HER-2/neu with the age, grade of the tumour and the ER and PR status. A multivariate analysis done by Huang et al has revealed that ER and PR were inversely related to HER-2/neu over-expression whereas tumour grade was positively associated with HER-2/neu over-expression. The size and grade represent important prognostic factors. Increase in size and grade are associated with poor prognosis. The NPI calculated using these two parameters and the lymph node status has been recognized as a good prognostic indicator in a number of multivariate analyses. ${ }^{12}$ The NPI of 15 out of $18 \mathrm{HER}-2 /$ neu positive breast cancers included in the study was more than 3.4 which reiterates the poor prognosis of the HER-2/neu positive status.

The efficacy of histological grade in predicting the status of HER-2/neu gene amplification was absolute in a study done by Sapino et al. ${ }^{13}$ All except one grade 1 tumour in our study were negative for HER-2/neu in keeping with the findings of the study done by Sapino et al.

Our study reveals that the four parameters assessed have a high negative predictive value in predicting the HER-2/neu status of breast carcinoma in the population studied. The positive predictive values are much lower for all four parameters.

We conclude from our study that a Nottingham Grade of 2 or 3, NPI of $>3.4$ and a Quick Score of 0 or 2 for ER and PR have a good negative predictive value in predicting the HER-2/neu status of breast carcinoma. Therefore the present study suggests that based on the Nottingham grade, $\mathrm{NPI}$ and the ER and PR status, the clinician should be able to indicate which breast carcinoma can be excluded from HER-2/neu assessment when it is necessary to select patients for HER$2 /$ neu assessment in a low resource setting.

\section{Acknowledgement}

Mrs. Dhammika Gunawardhane, Department of Pathology, Faculty of Medicine, Galle for immunohistochemical staining.

\section{References}

1. Cancer Incidence 1995. Cancer Registry. Sri Lanka: National Cancer Control Programme; 1995.

2. Fehm T, Jäger W, Krämer S, Sohn C, Solomayer E, Wallwiener D et al. Prognostic significance of serum HER-2 and CA 15-3 at the time of diagnosis of metastatic breast cancer. Anticancer Research. 2004; 24(3b):1987-92. 
3. Chen $\mathrm{CH}$, Lin YS, Lin CC, Yang YH, Ho YP, Tsai CC. Elevated serum levels of a c-erbB2 oncogene product in oral squamous cell carcinoma patients. Journal of Oral Pathology and Medicine. 2004; 33(10):589-94.

4. Miles DW. Update on HER-2 as a target for cancer therapy: Herceptin in the clinical setting. Breast Cancer Research [online]. 2001 Oct 11; 3(6):380-4.

5. Yuan P, Xu BH, Chu DT. Correlation between serum HER-2 oncoprotein and patients with breast cancer. Chinese Medical Science Journal. 2004 ; 19(3):212-5.

6. Prati R, Apple SK, He J, Gornbein JA, Chang HR. Histopathologic characteristics predicting HER-2/neu amplification in breast cancer. Breast Journal. 2005; 11(6):433-9.

7. Elston CW et al. Assessment of histological grade. In: Elston CW, Ellis IO editors. The Breast. Great Britain: Redwood Books Ltd 2000: 365-484.

8. Ellis IO, Bartlett J, Dowsett M, Humphreys S, Jasani B, Miller K et al. Best Practice No 176: Updated recommendation for HER-2 testing in the UK. Journal of Clinical Pathology. 2004; 57(3):233-7.

9. Leake R, Barnes D, Pinder S, Ellis I, Anderson L, Anderson T et al. Immunohistochemical detection of steroid receptors in breast cancer: a working protocol. UK Receptor Group, UK NEQAS, The Scottish Breast Cancer Pathology Group, and the Receptor and Biomarker Study Group of the EORTC. Journal of Clinical Pathology. 2000; 53(8):634-5.

10. Stuart JS. Traditional and newer pathologic factors. Journal of the National Cancer Institute Monographs; 2001 (No. 30).

11. Piccart-Gebhart MJ, Procter M, Leyland-Jones B, Goldhirsch A, Untch M, Smith I et al. Trastuzumab after adjuvant chemotherapy in HER2-positive breast cancer. New England Journal of Medicine. 2000; 353(16):1659-72.

12. Pathology Reporting of Breast Disease. NHS Breast Screening Programme. NHSBSP Publication; January 2005 (No. 58).

13. Sapino A, Coccorullo Z, Cassoni P, Ghisolfi G, Gugliotta P, Bongiovanni M et al. Which breast carcinomas need HER-2/neu gene study after immunohistochemical analysis? Results of combined use of antibodies against different c-erbB2 protein domains. Histopathology. 2003; 43(4):354-62. 